\title{
MHC Class I-Specific Inhibitory Receptors on CD8 T Cells - Impact on HIV-specific Activity Delphine Marsac ${ }^{1}$, Peggy Masdehors ${ }^{1}$, Isabelle Liberman ${ }^{1}$, Christophe Rapp², Jean-Paul Viard ${ }^{3}$ and Marie-Lise Gougeon* ${ }^{* \neq 1}$
}

\author{
Address: ${ }^{1}$ Department of Molecular Medicine, Institut Pasteur, Paris, ${ }^{2}$ Hôpital Militaire Bégin, St Mandé, France and ${ }^{3}$ Hôpital Necker, Paris, France \\ Email: Marie-Lise Gougeon* - mlgougeo@pasteur.fr \\ * Corresponding author ‡Presenting author
}

from 2005 International Meeting of The Institute of Human Virology
Baltimore, USA, 29 August - 2 September 2005
Published: 8 December 2005
Retrovirology 2005, 2(SuppI I):SIII doi:I0.1I86/1742-4690-2-SI-SIII

MHC class I-specific inhibitory receptors are expressed by a subset of memory-phenotype CD8+T cells. Similar to NK cells, MHC class I-specific inhibitory receptors might subserve on CD8 T cells an important negative control that participates to the prevention of autologous damage but may also contribute to viral escape.

We found that the expression of CD94 and KIRs is increased on CD8 T cells from HIV patients, and the accumulation of CD94+CD8+ T cells is driven by HIV replication.The expression of iNKR was found associated with a poor cytokine response (IFNg and TNFa) upon TCR triggering, not restored by IL-15.

The expression of CD94 and KIRs on HIV- and CMV-specific CD8 T cells was investigated with specific tetramer staining, and these NKR were barely detectable at the surface of virus-specific T cells, in contrast to CD85j. Characterization of the maturation stage of CD85jCD8 T cells, and of the impact of CD85j on CD8 T cell response upon TCR triggering with HIV-specific peptides is currently under investigation. 